\title{
POSTERIOR INTEROSSEOUS NEURITIS
}

\author{
W. J. W. Sharrard, Sheffield, England \\ From the Department of Orthopaedics, University of Sheffield
}

Spontaneous paralysis of the muscles supplied by the posterior interosseous nerve is rare. Benign tumours, usually lipomata, arising in the region of the elbow or at the upper end of the radius and causing nerve compression have been recorded (Moon and Marmor 1964; Barber, Bianco, Soule and MacCarty 1962) but they are uncommon, and Bowen and Stone (1966) have recorded an instance of paralysis caused by a ganglion at the elbow. This paper reports the findings at exploration in six patients who developed spontaneous paralysis of the posterior interosseous nerve. In two, a benign tumour at the level of the neck of the radius was found to be the cause, and histological examination showed them to be a calcified lipoma and a fibroma. In the other four patients traumatic neuritis of the posterior interosseous nerve was responsible. In each case recovery followed surgical decompression of the nerve.

\section{CASE REPORTS}

Case 1-In 1955 a man of thirty employed as a steel examiner noticed a lump on the outer side of the upper part of his right forearm. Four months later he noticed progressive inability to extend the fingers, and weakness of extension of the wrist. Eight months after this he was referred to hospital and was found to have a mass about 5 centimetres in diameter attached to and moving with the neck of the radius, with complete paralysis of all muscles supplied by the posterior interosseous nerve. Radiographs showed calcification in the tumour which was at first thought to be a chondroma that might be becoming malignant.

Operation-In January 1956 the lesion was explored. Attached to the neck of the radius there was a bi-lobed mass consisting of encapsulated fat with a bony-hard centre. The tumour was excised. The posterior interosseous nerve was not seen during the operation and was not looked for. Histological examination showed the tumour to be a calcified lipoma. Recovery of nerve function began ten weeks after the operation and was complete within nine months.

Case 2-In 1956 a woman of thirty-five began to notice weakness of extension of the right thumb and fingers. This progressed until she was unable to manage many of her household duties. On examination four months after the onset there was complete paralysis of the muscles supplied by the posterior interosseous nerve, but there was no other abnormality except for slight tenderness in the upper part of the forearm over the neck of the radius. Radiographic examination showed no abnormality.

Operation-In July 1956 the posterior interosseous nerve was explored. In the fibres of the supinator muscle it was displaced posteriorly by a firm tumour arising from the capsule of the elbow at the level of the neck of the radius and measuring 1.5 centimetres by 1 centimetre. The nerve was being compressed by the tumour and showed slight swelling beyond the site of compression. The tumour was removed. Histological examination showed it to be a simple fibroma. Recovery of wrist extensor power was observed two months after operation, and in due course finger and thumb extension was regained. Recovery was complete within nine months.

Case 3-In 1958 a boy of sixteen was being taught how to use a hammer in an awkward situation which required the elbow to be almost fully extended. Consequently, the elbow was repeatedly and sharply extended for an hour and a half. He noticed some pain in the elbow on that day. He continued work on the following two days and noticed nothing abnormal except continued aching pain in the elbow. After this he noticed impaired ability to straighten the fingers and thumb which became gradually worse during the next few days. Six months after the onset he consulted a neurologist who found complete paralysis of the muscles supplied by the posterior interosseous nerve. The elbow showed no abnormal or decreased mobility; there was no local tenderness and no palpable tumour in the region of the elbow.

Operation-In October 1958 the posterior interosseous nerve was explored through an antero-lateral incision. The radial nerve was identified in the lower part of the upper arm, where it was found to 
be normal. It was followed down to a point where it divided just above the level of the elbow into the posterior interosseous and superficial radial nerves. Just beyond this the posterior interosseous nerve lay in front of the capitulum, and immediately beyond this there was a soft neuroma of the nerve causing its expansion to a diameter about half as great again as normal. The swelling was of much the same character as that seen in traumatic ulnar neuritis. The nerve was slightly adherent to the front of the capsule of the elbow. On extension of the elbow, the capitulum bulged forward to produce compression of the nerve. It was thought that there might be some abnormality of the capitulum itself; it was therefore exposed but was found to be normal. The nerve was mobilised and the incision closed. Progressive recovery occurred in the muscles supplied by the posterior interosseous nerve and was complete after four years.

Case 4-In 1963 a left handed schoolboy of nine was snowballing for an hour. On the same evening he complained of pain in the left elbow when it was extended. Over the next three days the pain in the elbow improved but on the fourth day he began to notice weakness of extension of his fingers and thumb. This increased during the next four days and was associated with weakness of wrist extension. He was referred to hospital a few days later and was found to have 20 degrees painful limitation of extension of the left elbow, tenderness over the antero-lateral aspect of the joint and a complete posterior interosseous paralysis. The elbow was treated in a sling. A month later there was no change in the paralysis and electrical tests showed no response to faradism and a sluggish response to galvanism in the muscles supplied by the posterior interosseous nerve. Electromyographic examination of the extensor digiti minimi muscle showed spontaneous fibrillation at rest and no normal action potentials. Operation-The posterior interosseous nerve was explored two weeks later. The radial nerve was traced downwards to a point just above the capitulum where the superficial radial nerve branches off. Immediately beyond this the posterior interosseous nerve was covered by a thick band of fibrous tissue that was compressing it against the capsule of the elbow overlying the capitulum. The nerve was released and was seen to be thinned and grey in the region of constriction, and oedematous beyond this for a length of about 1 centimetre. Stimulation of the main radial nerve in the lower third of the forearm gave no response in any of the muscles supplied by the posterior interosseous nerve. The boy was discharged home with splints to maintain extension of the thumb and fingers.

One month later there was no change in the paralysis, but after another month recovery was noted in the extensor carpi ulnaris muscle. Two weeks later the finger and thumb extensors had recovered to power 3 (M.R.C.), and there was a complete return of function two months later.

Case 5-In July 1963 a stonemason of twenty-three fell on to his right hand with the elbow fully extended. His main symptoms were in the thumb where he had suffered a strain of the medial (ulnar collateral) ligament of the metacarpo-phalangeal joint, but he also had slight aching pain in the outer side of the elbow. He continued with his work, some of which involved hammering with the elbow almost fully extended for the next three days. He then woke up in the morning to find weakness of extension of his wrist, finger and thumb. Three days later he had complete paralysis of the posterior interosseous nerve. A cock-up splint was applied to the wrist and fingers. Four weeks later there was no sign of recovery in the posterior interosseous nerve. There was tenderness on pressure over the nerve anterior to the elbow joint. Six weeks after injury the posterior interosseous nerve was explored. It was found to be slightly oedematous and adherent to the front of the capsule of the elbow over the region of the capitulum. There was a soft neuroma distal to this point. Stimulation of the nerve gave a response in the extensors of the fingers and thumb. The nerve was mobilised and the incision closed. Two weeks later recovery was seen in the extensors of the thumb and fingers; one month after that, finger extension was recorded as power 4 and extensor pollicis brevis and longus as power 3 ; and six months later there was full power in all the muscles supplied by the posterior interosseous nerve. The medial ligament of the right thumb was repaired two months after this, and his ultimate function was full.

Case 6-In 1958 a man of thirty-nine engaged in secretarial work came to hospital complaining of pain in the right elbow and weakness of extension of the fingers and thumb. At the age of seven he had suffered an injury to the elbow for which an operation had been performed and there was a residual scar over the lateral epicondyle and just above it. A radiograph (Fig. 1) showed evidence of an old united supracondylar fracture with 30 degrees of varus deformity. Flexion and extension of the elbow were normal but there was paralysis of all the muscles supplied by the posterior interosseous nerve. There was no loss of sensibility to fine touch in the distribution of the superficial or radial nerve.

Three years earlier he had complained of pain in the right elbow. At that time there was no paralysis in any muscle supplied by the posterior interosseous nerve. He was given a short course of 
radiant heat and exercises for the elbow with relief of symptoms. Radiographs taken at that time were identical with those taken in 1958.

Operation-The lower end of the radial nerve and the proximal end of the posterior interosseous nerve and superficial radial nerve were explored. At a point 5 centimetres above the elbow the radial nerve was found to be grossly adherent and thickened. The thickening continued as far as the beginning of the posterior interosseous nerve but it did not involve the superficial radial nerve. Beyond the level of the elbow, the posterior interosseous nerve returned to normal size. The radial and posterior interosseous nerves were mobilised to lie more medially and the wound was closed. Recovery was

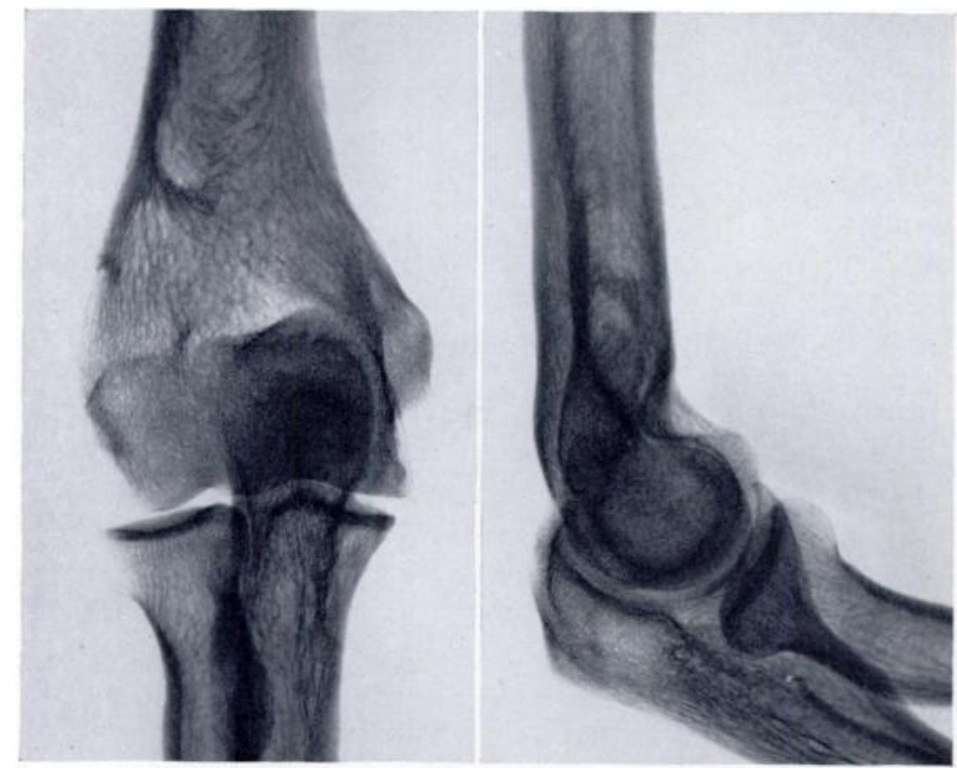

Fig. 1

Radiograph of the elbow in Case 6. There is evidence of an old supracondylar fracture of the humerus that has united with varus deformity.

first seen in the wrist extensor muscles six weeks later and in the extensors of the fingers two months later. Six months later there was normal power of wrist extension, extension of the fingers was generally about power 4 , and the thumb extensors and abductor pollicis longus muscle about power 3 . At the end of a year finger extension was normal and there was still slight weakness of abduction and extension of the thumb.

\section{DISCUSSION}

Paralysis of the posterior interosseous nerve associated with benign tumours has been fully discussed by Moon and Marmor (1964) and Barber et al. (1962). The two patients in whom a benign tumour was found in this series had clinical and operative findings very like those described in these papers. Traumatic posterior interosseous neuritis similar to that found in ulnar nerve paresis has not been recorded in the literature. Woltman and Learmonth (1934) described five cases of spontaneous paralysis but exploration was performed in only one instance, in which the nerve was found to take an abnormal course. Weinberger (1939) described two cases which he attributed to inflammation of the bicipito-radial and interosseous bursae of the forearm, but as exploration was not performed his hypothesis remains unproven. In three patients in this series the clinical and operative findings suggested that acute or sub-acute hyperextension trauma to the elbow had ruptured some fibres of the anterior capsule. Secondarily to this, the nerve had become adherent and had suffered stretching or compression, or both, with delayed paralysis of the muscles supplied by the posterior interosseous nerve. In the patient whose nerve was explored six weeks after the onset of symptoms (Case 5) the subsequent recovery suggested that the nerve had sustained a mixture of neurapraxia and 
axonotmesis. In the patients explored at ten weeks and three months, subsequent recovery was consistent with axonotmesis that showed recovery by downgrowth of axons. The fourth patient (Case 6), who had suffered a cubitus varus deformity from a childhood fracture, presented a clinical history and findings comparable with those found in the ulnar nerve in long standing cubitus valgus (Platt 1928). Recovery in this case was less complete. The fact that an operation had been performed on the lateral side of the elbow in childhood may have been an additional factor in producing adhesion of the nerve to its surroundings. The conclusions to be drawn from this small series are that persistent paralysis of the posterior interosseous nerve warrants operative exposure to determine the cause of the paralysis, which may be associated with a tumour or with adherence to the elbow joint and compression producing a posterior interosseous neuritis. Relief of the nerve from compression, if performed early enough, should bring complete recovery.

\section{SUMMARY}

1. Six patients suffering from spontaneous posterior interosseous paralysis are described.

2. Two were due to benign tumour and four due to traumatic neuritis, three of which were associated with minor hyperextension injuries to the elbow joint and one with long standing cubitus varus.

3. Surgical exploration was performed in each patient with recovery of nerve function.

\section{REFERENCES}

Barber, K. W., Jun., Bianco, A. J., Jun., Soule, E. H., and MacCarty, C. S. (1962): Benign Extraneural Soft-Tissue Tumors of the Extremities Causing Compression of Nerves. Journal of Bone and Joint Surgery, 44-A, 98.

Bowen, T. L., and Stone, K. H. (1966): Posterior Interosseous Nerve Paralysis Caused by a Ganglion at the Elbow. Journal of Bone and Joint Surgery, 48-B, 774.

Moon, N., and MARmor, L. (1964): Parosteal Lipoma of the Proximal Part of the Radius. Journal of Bone and Joint Surgery, 46-A, 608.

Platt, H. (1928): On the Peripheral Nerve Complications of Fractures and Dislocations of the Elbow. In The Robert Jones Birthday Volume, p. 195. London: Humphrey Milford Oxford University Press.

Weinberger, L. M. (1939): Non-traumatic Paralysis of the Dorsal Interosseous Nerve. Surgery, Gynecology and Obstetrics, 69, 358.

Woltman, H. W., and Learmonth, J. R. (1934): Progressive Paralysis of the Nervous Interosseous Dorsalis. Brain, 57, 25. 